\author{
ANNALES \\ POLONICI MATHEMATICI \\ XLVII (1987)
}

\title{
Generic properties of some iterative functional equations
}

by WITOLD J ARCZYK (Katowice)

\begin{abstract}
In the paper equations of the form (1) are studied, where the functions $h$ are monotonic as functions of the second variable. It is shown that existence and uniqueness as well as continuous dependence of continuous solutions of equations (1) are generic properties. The density of the set of all equations (1) having no continuous solutions is also proved.
\end{abstract}

In papers [4] and [5] some properties of equations of the form

$$
\varphi(x)=h(x, \varphi[f(x)])
$$

have been proved to be generic in suitable spaces $\mathscr{H}$ and $\mathscr{F} \times \mathscr{H}$ of given functions $h$ and pairs $(f, h)$, respectively. Here we shall discuss the same properties taking into account only equations (1) with real functions $h$ which are monotonic with respect to the second variable. Such assumptions may be found, for example, in papers [1], [7], and [11]. Observe also that the linear functional equation

$$
\varphi[f(x)]=g(x) \varphi(x)+F(x)
$$

is of the form (1) with a function $h$ which, as a function of the second variable, is linear and thus monotonic. Some methods used in this paper are patterned upon those presented by J. Myjak (cf. [10], Theorem 1.2).

In the sequel, given topological spaces $X$ and $Y$ we shall denote by $\mathscr{C}(X, Y)$ the set of all functions mapping $X$ continuously into $Y$. We shall treat it as a topological space with compact-open topology (cf. [9], § 44).

Remark 1. If $X$ is a topological space and $(Y, d)$ is a metric space, then the family of all sets of the form

$$
\left\{f \in \mathscr{C}(X, Y): d\left(f(x), f_{0}(x)\right)<\varepsilon, x \in C\right\},
$$

where $C$ is a compact subset of $X$ and $\varepsilon$ is a positive number, is a basis at $f_{0} \in \mathscr{C}(X, Y)$.

We omit a simple proof of this remark.

In the whole paper we shall assume that $(X, \varrho)$ is a metric space and $\xi$ is 
a fixed point in $X$. Denote by $\mathscr{F}$ the subset of $\mathscr{C}(X, X)$ consisting of all functions satisfying the inequality

$$
\varrho(f(x), \xi) \leqslant \gamma_{f}(\varrho(x, \xi)), \quad x \in X,
$$

where $\gamma_{f}$ is an increasing and right-continuous real function defined on an interval $I$ containing the origin, and $\gamma_{f}(t)<t$ for every $t \in I \backslash\{0\}$.

\section{Remark 2. If}

(2) the ball $\left\{x \in X: \varrho(x, \xi) \leqslant \varrho\left(x_{0}, \xi\right)\right\}$ is compact for every $x_{0} \in X$, then $\mathscr{F}$ is the set of all $f \in \mathscr{C}(X, X)$ such that $f(\xi)=\xi$ and

$$
\varrho(f(x), \xi)<\varrho(x, \xi), \quad x \in X \backslash\{\xi\}
$$

(cf. [2], Theorem 3.3). If condition (2) holds, then $X$ is a separable locally compact space (cf. [5], Lemma 3).

Remark 3 (cf. [4], Remark 1). If $f \in \mathscr{F}$ then the sequence $\left(f^{k}: k \in N\right)\left({ }^{1}\right)$ converges to $\xi$ uniformly on every compact subset of $X$ and, in particular, $\xi$ is the unique fixed point of $f$.

Fix a real number $\eta$ and denote by $\Phi$ the set of all elements of $\mathscr{G}(X, R)$ taking the value $\eta$ at the point $\xi$. Clearly, if $f \in \mathscr{F}, h \in \mathscr{C}(X \times \boldsymbol{R}, \boldsymbol{R})$ and $\varphi \in \Phi$ is a solution of equation (1), then

$$
h(\xi, \eta)=\eta \text {. }
$$

Therefore it is of interest to consider the set $\mathscr{H}$ of all functions $h \in$ $\mathscr{C}(X \times \boldsymbol{R}, \boldsymbol{R})$ satisfying equality (3). Here we shall be mainly interested in those elements $h$ of $\mathscr{H}$ for which the function $h(x, \cdot)$ is monotonic for every $x \in X$ (the kind of monotonicity of $h(x, \cdot)$ may depend on $x$ ). The set of all these functions will be denoted by $\mathscr{M}$.

Remark 4. If $X$ is a separable locally compact space, then $\mathscr{M}$ is a topologically complete space (metrizable by the metric of uniform convergence on compact sets). If $X$ is a topologically complete space satisfying condition (2), then $\mathscr{F}, \mathscr{M}$, and $\mathscr{F} \times \mathscr{M}$ are topologically complete spaces (metrizable by the metric of uniform convergence on compact sets).

Proof. Replacing above $\mathscr{M}$ by $\mathscr{H}$ we get exactly the contents of [5], Remarks 1 and 2. To get the assertion it is enough to observe that $\mathscr{M}$ and $\mathscr{F} \times \mathscr{M}$ are closed subsets of $\mathscr{H}$ and $\mathscr{F} \times \mathscr{H}$, respectively.

We start with the following lemma.

Lemma 1. Suppose that $h, h^{*} \in \mathscr{M}$ and, for every $x$ from a neighbourhood of $\xi$, the functions $h(x, \cdot)$ and $h^{*}(x, \cdot)$ are simultaneously increasing or decreasing. Then for every positive number $\varepsilon$ there exists a function $h^{\prime} \in \mathscr{M}$ such that

$$
\left|h(x, y)-h^{\prime}(x, y)\right|<\varepsilon, \quad(x, y) \in X \times R,
$$

(1) For every nonnegative integer $k, f^{k}$ denotes the $k$ th iterate of $f$. 
and

$$
h^{\prime}(x, y)=h^{*}(x, y)
$$

for every $(x, y)$ from a neighbourhood of $(\xi, \eta)$.

Proof. Let $U$ be a neighbourhood of $\xi$ such that for $x \in U$ the functions $h(x, \cdot)$ and $h^{*}(x, \cdot)$ are simultaneously increasing or decreasing. Fix a positive number $\varepsilon$ and choose an open neighbourhood $V \subset U$ of $\xi$ and a positive number $\delta$ in such a manner that

$$
|h(x, y)-h(x, \eta)|<\varepsilon / 2, \quad x \in V,|y-\eta| \leqslant \delta,
$$

and

$$
\left|h^{*}(x, y)-h(x, \eta)\right|<\varepsilon / 2, \quad x \in V,|y-\eta| \leqslant \delta .
$$

Fix a closed neighbourhood $W$ of $\xi$ contained in $V$. On account of Urysohn's Lemma (cf. [8], $\S 14$, IV) there exists a continuous function $p$ : $X \rightarrow[0,1]$ such that $p(x)=0$ for $x \in X \backslash V$ and $p(x)=1$ for $x \in W$. Define a function $F: X \times \boldsymbol{R} \rightarrow \boldsymbol{R}$ by

$$
F(x, y)= \begin{cases}\eta-\delta p(x) & \text { if } x \in X \text { and } y<\eta-\delta p(x) \\ y & \text { if } x \in X \text { and }|y-\eta| \leqslant \delta p(x) \\ \eta+\delta p(x) & \text { if } x \in X \text { and } y>\eta+\delta p(x)\end{cases}
$$

Since the function $F$ is continuous in each variable separately and the function $F(x, \cdot)$ is increasing for every $x \in X$, the function $F$ is continuous. Moreover,

$$
|F(x, y)-\eta| \leqslant \delta p(x), \quad(x, y) \in X \times R
$$

and so (cf. (4))

$$
|h(x, F(x, y))-h(x, \eta)|<\varepsilon / 2, \quad(x, y) \in X \times R .
$$

The function $h_{0}: X \times \boldsymbol{R} \rightarrow \boldsymbol{R}$ defined by

$$
h_{0}(x, y)=p(x) h^{*}(x, y)+(1-p(x)) h(x, \eta)
$$

is an element of $\mathscr{M}$ and it follows from the assumption of the lemma that, for every $x \in X$, the functions $h_{0}(x, \cdot)$ and $h(x, \cdot)$ are simultaneously increasing or decreasing. Finally, in view of (6) and (5),

$$
\begin{array}{r}
\left|h_{0}(x, F(x, y))-h(x, \eta)\right|=p(x)\left|h^{*}(x, F(x, y))-h(x, \eta)\right|<\varepsilon / 2, \\
(x, y) \in X \times \boldsymbol{R} .
\end{array}
$$

Now define a function $h^{\prime}: X \times \boldsymbol{R} \rightarrow \boldsymbol{R}$ by

$$
h^{\prime}(x, y)=h(x, y)-h(x, F(x, y))+h_{0}(x, F(x, y)) \text {. }
$$

Since, by the definition of $F$, for every $x \in X$ the functions 
$h(x, \cdot)-h(x, F(x, \cdot))$ and $h(x, \cdot)$ are simultaneously increasing or decreasing and the same is true for the functions $h_{0}(x, \cdot)$ and $h(x, \cdot)$, the function $h^{\prime}$ is an element of $\mathscr{M}$. Moreover, on account of inequalities (7) and (8),

$$
\left|h(x, y)-h^{\prime}(x, y)\right|<\varepsilon, \quad(x, y) \in X \times \boldsymbol{R} .
$$

If $x \in W$ and $|y-\eta| \leqslant \delta$, then $p(x)=1$ and $F(x, y)=y$, whence

$$
h^{\prime}(x, y)=h_{0}(x, y)=h^{*}(x, y),
$$

and the lemma is proved.

Let us denote by $\mathscr{H}_{0}$ and $\mathscr{M}_{0}$ the subsets of $\mathscr{H}$ and $\mathscr{M}$, respectively, consisting of all functions taking the value $\eta$ in a neighbourhood of the point $(\xi, \eta)$.

The following corollary is an immediate consequence of Lemma 1 and Remark 1.

Corollary 1. The set $\mathscr{M}_{0}$ is dense in $\mathscr{M}$.

For any $(f, h) \in \mathscr{F} \times \mathscr{H}$ define a mapping $T(f, h): \Phi \rightarrow \Phi$ by

$$
T(f, h)(\varphi)(x)=h(x, \varphi[f(x)]), \quad x \in X .
$$

The next lemma follows directly from [5], Lemmas 4 and 5.

Lemma 2. Let $C$ be a compact neighbourhood of $\xi$ such that $f(C) \subset C$ for any $f \in \mathscr{F}$. Then, for every $\left(f_{0}, h_{0}\right) \in \mathscr{F} \times \mathscr{H}_{0}$ and any positive integer $k$, there exist neighbourhoods $\mathscr{U}_{c}\left(f_{0}, h_{0}, 1 / k\right) \subset \mathscr{F}$ and $\mathscr{V}_{c}\left(f_{0}, h_{0}, 1 / k\right) \subset \mathscr{H}$ of $f_{0}$ and $h_{0}$, respectively, such that, for every pair $(f, h)$ belonging to the set

$$
\mathscr{R}(C)=\bigcap_{k=1}^{\infty} \bigcup_{\left(f^{\prime}, h^{\prime}\right) \in \mathbb{F}^{\prime}, \mathscr{H}_{0}} \mathscr{U}_{c}\left(f^{\prime}, h^{\prime}, 1 / k\right) \times \mathscr{V}_{c}\left(f^{\prime}, h^{\prime}, 1 / k\right),
$$

equation (1) has exactly one solution $\varphi \in \Phi$ and for every $\varphi_{0} \in \Phi$ the sequence $\left(T(f, h)^{k}\left(\varphi_{0}\right): k \in N\right)$ of successive approximations converges to $\varphi$ uniformly on every compact subset of $X$.

Now we can prove an analog of Theorem 1 from paper [5].

THEOREM 1. Suppose that the point $\xi$ has a compact neighbourhood in $X$. Then the set of all pairs $(f, h) \in \mathscr{F} \times \mathscr{M}$ such that equation (1) has exactly one solution $\varphi \in \Phi$ and for every $\varphi_{0} \in \Phi$ the sequence $\left(T(f, h)^{k}\left(\varphi_{0}\right): k \in N\right)$ of successive approximations converges to $\varphi$ uniformly on every compact subset of $X$ is residual in $\mathscr{F} \times \mathscr{M}$.

Proof. Since there exists a compact neighbourhood of $\xi$, we can find a compact ball $C$ centered at $\xi$. Observe that $f(C) \subset C$ for any $f \in \mathscr{F}$. In view of Lemma 2, the set

$$
\mathscr{R}^{*}(C)=\bigcap_{k=1}^{\infty} \bigcup_{\left(f^{\prime}, h^{\prime}\right) \in \mathscr{F} \times \mathscr{M}_{0}} \mathscr{U}_{C}\left(f^{\prime}, h^{\prime}, 1 / k\right) \times\left[\mathscr{V}_{c}\left(f^{\prime}, h^{\prime}, 1 / k\right) \cap \mathscr{M}\right]
$$

is a $G_{\delta}$ subset of the space $\mathscr{F} \times \mathscr{M}$. Moreover, since $\mathscr{M}_{0}$ is a dense subset of 
(cf. Corollary 1) and $\mathscr{F} \times \mathscr{M}_{0} \subset \mathscr{R}^{*}(C), \mathscr{R}^{*}(C)$ is also a dense set. Consequently, the set $\mathscr{R}^{*}(C)$ is residual in $\mathscr{F} \times \mathscr{M}$. Clearly, $\mathscr{R}^{*}(C) \subset \mathscr{R}(C)$, thus the theorem follows from Lemma 2.

In a completely similar way (making use of Lemma 2 and Corollary 1 or [4], Lemmas 4 and 2) we get the following analog of Theorem 1 from paper [4].

THEOREM 2. Suppose that the point $\xi$ has a compact neighbourhood in $X$. Then, for every $f \in \mathscr{F}$, the set of all functions $h \in \mathscr{M}$ such that equation (1) has exactly one solution $\varphi \in \Phi$ and for every $\varphi_{0} \in \Phi$ the sequence $\left(T(f, h)^{k}\left(\varphi_{0}\right): k \in N\right)$ of successive approximations converges to $\varphi$ uniformly on every compact subset of $X$ is residual in $\mathscr{M}$.

Now we shall study the set of all "pathological" equations (1) having no solution in the class $\Phi$ (cf. [4], Theorem 2, and [5], Theorem 3). First we shall prove two lemmas.

Lemma 3. The set of all functions $h \in \mathscr{M}$ for which there exists a neighbourhood $U$ of $\xi$ such that all functions $h(x, \cdot), x \in U$, are simultaneously increasing or decreasing is dense in $\mathscr{M}$.

Proof. Fix a function $h_{0} \in \mathscr{M}$ without the property required in the lemma. Then there exist sequences $\left(x_{n}: n \in N\right)$ and $\left(x_{n}^{\prime}: n \in N\right)$ of elements of $X$ converging to $\xi$ such that, for every $n \in N$, the function $h_{0}\left(x_{n}, \cdot\right)$ is decreasing and the function $h_{0}\left(x_{n}^{\prime}, \cdot\right)$ is increasing. Hence, in view of the continuity of $h_{0}$, the function $h_{0}(\xi, \cdot)$ is constant, i.e.,

$$
h_{0}(\xi, y)=\eta, \quad y \in R \text {. }
$$

Fix a neighbourhood $\mathscr{U}$ of $h_{0}$. By Remark 1 we can assume that

$$
\mathscr{U}=\left\{h \in \mathscr{M}:\left|h(x, y)-h_{0}(x, y)\right|<\varepsilon, x \in C,|y-\eta| \leqslant a\right\},
$$

where $C$ is a compact subset of $X$, and $a$ and $\varepsilon$ are positive numbers. According to (10) we can find an open neighbourhood $V$ of $\xi$ such that

$$
\left|h_{0}(x, y)-\eta\right|<\varepsilon, \quad x \in V,|y-\eta| \leqslant a .
$$

Let $W \subset V$ be a closed neighbourhood of $\xi$. On account of Urysohn's Lemma there exists a function $p \in \mathscr{C}(X,[0,1])$ such that $p(x)=0$ for $x \in W$ and $p(x)=1$ for $x \in X \backslash V$. The function $h: X \times R \rightarrow R$ defined by

$$
h(x, y)=p(x) h_{0}(x, y)+(1-p(x)) \eta
$$

belongs to $\mathscr{M}$. Moreover, for every $x \in W$, the function $h(x, \cdot)$ is constant, and, in view of (11) and the properties of $p$,

$$
\left|h(x, y)-h_{0}(x, y)\right|=(1-p(x))\left|h_{0}(x, y)-\eta\right|<\varepsilon, \quad x \in X,|y-\eta| \leqslant a .
$$

In particular, $h \in \mathscr{U}$ and the lemma is proved. 
Lemma $4\left({ }^{2}\right)$. Suppose that $f \in \mathscr{F}$ and the set $\left\{f^{k}\left(x_{0}\right): k \in N\right\}$ is infinite for an $x_{0} \in X$. Then, for any $a \in\{-1,1\}$, there exists a function $g \in \mathscr{C}(X, R)$ such that:

(i) $g(\xi)=(1-a) \eta$;

(ii) if $h \in \mathscr{H}$ and $h(x, y)=g(x)+$ ay for every $(x, y)$ from a neighbourhood of $(\xi, \eta)$, then equation (1) has no solution in the class $\Phi$.

Proof. Let $\left(y_{k}: k \in N\right)$ be a sequence of real numbers such that

$$
\lim _{k \rightarrow \infty} y_{k}=0
$$

and

(13) the series $\sum_{k=1}^{\infty} y_{k}$ diverges.

Put $F=\left\{f^{k}\left(x_{0}\right): k \in N\right\} \cup\{\xi\}$ and define a function $\bar{g}: F \rightarrow R$ by

$$
\bar{g}(\xi)=(1-a) \eta \quad \text { and } \quad \bar{g}\left[f^{k}\left(x_{0}\right)\right]=a^{k} y_{k}+(1-a) \eta, \quad k \in N .
$$

Since $F$ is infinite and $f \in \mathscr{F}$, we have

$$
f^{k}\left(x_{0}\right) \neq \xi, \quad k \in N
$$

and

$$
f^{i}\left(x_{0}\right)=f^{j}\left(x_{0}\right) \quad \text { implies } \quad i=j, i, j \in N .
$$

Thus the definition of $\bar{g}$ is correct. By Remark 3, $\xi$ is the unique accumulation point of the set $F$. Hence, and from (12), it follows that $F$ is a closed subset of $X$ and the function $\bar{g}$ is continuous. Using the Dugundji Extension Theorem (cf. [3], Chapter II, Theorem 3.1) we can find a function $g \in \mathscr{C}(X, R)$ such that $\left.g\right|_{F}=\bar{g}$ and, by (14), condition (i) holds.

Now, passing to the proof of (ii), suppose that $h \in \mathscr{H}$ and

$$
h(x, y)=g(x)+a y, \quad(x, y) \in U \times V,
$$

where $U$ and $V$ are neighbourhoods of $\xi$ and $\eta$, respectively.

Suppose, contrary to our claim, that a function $\varphi \in \Phi$ satisfies equation (1). Since the function $\varphi$ is continuous at $\xi$ and $\varphi(\xi)=\eta$, there exists a neighbourhood $W$ of $\xi$ such that $W \subset U$ and $\varphi(W) \subset V$. We can also assume that $f(W) \subset W$. If $x \in W$, then $(x, \varphi[f(x)]) \in U \times V$ and, in virtue of (15),

$$
\varphi(x)=h(x, \varphi[f(x)])=g(x)+a \varphi[f(x)], \quad x \in W .
$$

By Remark 3, $f^{n}\left(x_{0}\right) \in W$ for a positive integer $n$. It follows from (16) (cf. [6],

$\left({ }^{2}\right)$ This lemma remains true (with no changes in the proof) if $R$ is replaced by an arbitrary nontrivial normed space. 
Chapter II, Theorem 2.11) that the series

$$
\sum_{k=1}^{\infty} a^{k}\left(g\left[f^{k+n}\left(x_{0}\right)\right]-(1-a) \eta\right)
$$

converges. On the other hand, in view of (14),

$$
\sum_{k=1}^{\infty} a^{k}\left(g\left[f^{k+n}\left(x_{0}\right)\right]-(1-a) \eta\right)=\sum_{k=1}^{\infty} a^{k} a^{k+n} y_{k+n}=a^{n} \sum_{k=n+1}^{\infty} y_{k},
$$

which contradicts property (13). Thus the proof is complete.

TheOREM 3. Suppose that $f \in \mathscr{F}$ and the set $\left\{f^{k}\left(x_{0}\right): k \in N\right\}$ is infinite for an $x_{0} \in X$. Then the set of all functions $h \in \mathscr{M}$ for which equation (1) has no solution in the class $\Phi$ is dense in $\mathscr{M}$.

Prool. Fix a function $h_{0} \in \mathscr{M}$ and its neighbourhood $\mathscr{U}$. We can assume (cf. Remark 1) that

$$
\mathscr{U}=\left\{h \in \mathscr{M}:\left|h(x, y)-h_{0}(x, y)\right|<\varepsilon, x \in C,|y-\eta| \leqslant a\right\},
$$

where $C$ is a compact subset of $X$, and $a$ and $\varepsilon$ are positive numbers. On account of Lemma 3 , there exist a function $h \in \mathscr{M}$ and a neighbourhood $U$ of $\xi$ such that

$$
\left|h(x, y)-h_{0}(x, y)\right|<\varepsilon / 2, \quad x \in C,|y-\eta| \leqslant a,
$$

and all the functions $h(x, \cdot), x \in U$, are simultaneously increasing or decreasing.

Put $a=-1$ if the functions $h(x, \cdot), x \in U$, are decreasing and $a=1$ if the functions $h(x, \cdot), x \in U$, are increasing. Let $g \in \mathscr{C}(X, R)$ be a function satisfying conditions (i) and (ii) in Lemma 4. The function $h^{*}: X \times \boldsymbol{R} \rightarrow \boldsymbol{R}$ defined by

$$
h^{*}(x, y)=g(x)+a y
$$

is an element of $\mathscr{M}$. In view of Lemma 1 there exists a function $h^{\prime} \in \mathscr{M}$ such that

$$
\left|h(x, y)-h^{\prime}(x, y)\right|<\varepsilon / 2, \quad(x, y) \in X \times R,
$$

and

$$
h^{\prime}(x, y)=h^{*}(x, y)=g(x)+a y
$$

for every $(x, y)$ from a neighbourhood of $(\xi, \eta)$. By (ii), the equation

$$
\varphi(x)=h^{\prime}(x, \varphi[f(x)])
$$

has no solution in the class $\Phi$, and, in virtue of (17) and (18), $h^{\prime} \in \dot{U}$. This ends the prool.

It has been proved (cf. [5], Lemma 7) that if $X$ is a convex subset of a 
normed space, then the set of all functions $f \in \mathscr{F}$ such that the set $\left\{f^{k}\left(x_{0}\right): k \in N\right\}$ is infinite for an $x_{0} \in X$ is dense in $\mathscr{F}$. Hence, and from Theorem 3, we deduce the following result, which is an analog of [5], Theorem 3.

THEOREM 4. Suppose that $X$ is a convex subset of a normed space. Then the set of all pairs $(f, h) \in \mathscr{F} \times \mathscr{M}$ for which equation (1) has no solution in the class $\Phi$ is dense in $\mathscr{\mathcal { F }} \times .11$.

The results given below concern the problem of the continuous dependence of continuous solutions of equation (1). They are immediate consequences of those given in [5] (cf. [5], Theorem 2 and Corollary). If $(f, h) \in$ $\mathscr{F} \times \mathscr{M}$ and equation (1) has exactly one solution in the class $\Phi$, then we shall denote it by $\varphi_{f, h}$. By $\Phi_{C}$, where $C$ is a subset of $X$, we shall mean the set of all restrictions of functions from $\Phi$ to the set $C$.

THEOREM 5. Let $C$ be a compact neighbourhood of $\xi$ such that $f(C) \subset C$ for any $f \in \mathscr{F}$ and let $\mathscr{R}^{*}(C)$ be given by (9). Then the map $\Lambda_{C}: \mathscr{R}^{*}(C) \rightarrow \Phi_{C}$ given by

$$
\Lambda_{C}(f, h)=\left.\varphi_{f, h}\right|_{C}
$$

is well defined and continuous in $\mathscr{R}^{*}(C)$ (which is a residual subset of $\mathscr{F} \times \mathscr{M}$ ).

Corollary 2. Let $\left(C_{n}: n \in N\right)$ be a sequence of compact neighbourhoods of $\xi$ such that $X=\bigcup_{n=1}^{\infty} C_{n}, C_{n} \subset \operatorname{Int} C_{n+1}, n \in N$, and $f\left(C_{n}\right) \subset C_{n}$ for every $f \in \mathscr{F}$ and $n \in N$. Then the map $A: \bigcap_{n=1}^{\infty} \mathscr{R}^{*}\left(C_{n}\right) \rightarrow \Phi$ given by

$$
\Lambda(f, h)=\varphi_{f, h}
$$

is well defined and continuous in $\bigcap_{n=1}^{\infty} \mathscr{R}^{*}\left(C_{n}\right)$ (which is a residual subset of $\mathscr{F} \times \mathscr{M})$.

Note also that suitable versions of Theorem 3 and Corollary from paper [4] can be proved.

\section{References}

[1] K. Ba ron, On the continuous solutions of a non-linear functional equation of the first order, Ann. Polon. Math. 28 (1973), 201-205:

[2] -, Functional equations of infinite order, Prace Naukowe Uniwersytetu Śląskiego $\mathrm{nr} 265$, Uniwersytet Ślaski, Katowice 1978.

[3] C. Bessaga and A. Pełczyński, Selected topics in infinite-dimensional topology, Monografie Mat. 58, PWN, Warszawa 1975.

[4] W. Jarczyk, Generic properties of nonlinear functional equations, Aequationes Math. 26 (1983), 40-53. 
[5] W. Jarczyk, Existence and uniqueness of continuous solutions of nonlinear functional equations are generic properties, Ann. Math. Silesianae (to appear).

[6] M. Kuczma, Functional equations in a single variable, Monografie Mat. 46, PWN, Warszawa 1968.

[7] -, On the number of continuous solutions of a functional equation, Prace Naukowe Uniwersytetu Śląskiego nr 158, Prace Mat. 7 (1977), 51-55.

[8] K. Kuratowski, Topology, Vol. I, Academic Press, New York-London-TorontoSydney-San Francisco and Polish Scientific Publishers, Warszawa 1966.

[9] -, Topology, Vol. II, Academic Press, New York-London-Toronto-Sydney-San Francisco and Polish Scientific Publishers, Warszawa 1968.

[10] J. Myjak, Orlicz type category theorems for functional and differential equations, Dissert. Math. 206 (1983).

[11] A. Pelczar, On the extremal solutions of a functional equation, Zeszyty Naukowe Uniwersytetu Jagiellońskiego nr 55, Prace Mat. 7 (1962), 9-11.

AMS SUBJECT CLASSIFICATION (1980): PRIMARY 39B10

Reçu par la Rédaction le 1985.03.11 